\title{
Holes in graphs
}

\author{
Yuejian Peng \\ Department of Mathematics and Computer Science \\ Emory University, Atlanta, USA \\ peng@mathcs . emory. edu \\ Vojtech Rödl* \\ Department of Mathematics and Computer Science \\ Emory University, Atlanta, USA \\ rodl@mathcs . emory . edu \\ Andrzej Ruciński ${ }^{\dagger}$ \\ Department of Discrete Mathematics \\ Adam Mickiewicz University, Poznań, Poland \\ rucinski@amu.edu.pl \\ Submitted: November 7, 2000; Accepted: October 14, 2001. \\ MR Subject Classifications: 05C35
}

\begin{abstract}
The celebrated Regularity Lemma of Szemerédi asserts that every sufficiently large graph $G$ can be partitioned in such a way that most pairs of the partition sets span $\epsilon$-regular subgraphs. In applications, however, the graph $G$ has to be dense and the partition sets are typically very small. If only one $\epsilon$-regular pair is needed, a much bigger one can be found, even if the original graph is sparse. In this paper we show that every graph with density $d$ contains a large, relatively dense $\epsilon$-regular pair. We mainly focus on a related concept of an $(\epsilon, \sigma)$-dense pair, for which our bound is, up to a constant, best possible.
\end{abstract}

\section{Introduction}

Szemerédi's Regularity Lemma is one of the most powerful tools in extremal graph theory. It guarantees an $\epsilon$-regular partition of every graph $G$ with $n$ vertices, but the size of each

\footnotetext{
*Research supported by NSF grant DMS 9704114.

${ }^{\dagger}$ Research supported by KBN grant 2 P03A 032 16. Part of this research was done during the author's visit to Emory University.
} 
$\epsilon$-regular pair is at most $n / T$, where $T$ is the tower of 2 's of height $(1 / \epsilon)^{\frac{1}{16}}([4])$. However, in some applications, only one pair is needed. That was already observed and explored by Komlós (see [8]) and Haxell [6]. The goal of this paper is to estimate the size of the largest such pair that can be found in any graph of given size and density. The density may decay to 0 with $n \rightarrow \infty$.

The density of a bipartite graph $G=\left(V_{1}, V_{2}, E\right)$ is defined as

$$
d(G)=\frac{|E|}{\left|V_{1}\right|\left|V_{2}\right|}
$$

and the density of a pair $\left(U_{1}, U_{2}\right)$, where $U_{1} \subseteq V_{1}$ and $U_{2} \subseteq V_{2}$, is defined as

$$
d\left(U_{1}, U_{2}\right)=\frac{e\left(U_{1}, U_{2}\right)}{\left|U_{1}\right|\left|U_{2}\right|}
$$

where $e\left(U_{1}, U_{2}\right)$ is the number of edges of $G$ with one endpoint in $U_{1}$ and the other in $U_{2}$.

Definition 1.1 Let $G=\left(V_{1}, V_{2}, E\right)$ be a bipartite graph and $0<\epsilon<1$. A pair $\left(U_{1}, U_{2}\right)$, where $U_{1} \subseteq V_{1}$ and $U_{2} \subseteq V_{2}$, is called $\epsilon$-regular if for every $W_{1} \subseteq U_{1}$ and $W_{2} \subseteq U_{2}$ with $\left|W_{1}\right| \geq \epsilon\left|U_{1}\right|$ and $\left|W_{2}\right| \geq \epsilon\left|U_{2}\right|$, we have

$$
(1-\epsilon) d\left(U_{1}, U_{2}\right) \leq d\left(W_{1}, W_{2}\right) \leq(1+\epsilon) d\left(U_{1}, U_{2}\right)
$$

Our first result states that in every bipartite graph one can find a reasonably large and relatively dense $\epsilon$-regular pair.

Theorem 1.1 Let $0<\epsilon, d<1$. Then every bipartite graph $G=\left(V_{1}, V_{2}, E\right)$ with $\left|V_{1}\right|=$ $\left|V_{2}\right|=n$ and $d(G)=d$ contains an $\epsilon$-regular pair $\left(U_{1}, U_{2}\right)$ with density not smaller than $\left(1-\frac{\epsilon}{3}\right) d$ and $\left|U_{1}\right|=\left|U_{2}\right| \geq \frac{n}{2} d^{c / \epsilon^{2}}$, where $c$ is an absolute constant.

The constant $c$ in Theorem 1.1 is determined by inequality (40). For instance, one can take $c=50$.

In most applications the whole strength of $\epsilon$-regular pairs is not used. Instead, it is only required that $d\left(W_{1}, W_{2}\right)$ is not much smaller than $d\left(U_{1}, U_{2}\right)$ whenever $W_{1} \subseteq U_{1}$ and $W_{2} \subseteq U_{2}$ are large enough. This observation leads to the following definition.

Definition 1.2 Let $G=\left(V_{1}, V_{2}, E\right)$ be a bipartite graph and $0<\epsilon, \sigma<1$. A pair $\left(U_{1}, U_{2}\right)$, where $U_{1} \subseteq V_{1}$ and $U_{2} \subseteq V_{2}$, is called $(\epsilon, \sigma)$-dense if for every $W_{1} \subseteq U_{1}$ and $W_{2} \subseteq U_{2}$ with $\left|W_{1}\right| \geq \epsilon\left|U_{1}\right|$ and $\left|W_{2}\right| \geq \epsilon\left|U_{2}\right|$, we have $e\left(W_{1}, W_{2}\right) \geq \sigma\left|W_{1}\right|\left|W_{2}\right|$. The graph $G$ itself is called $(\epsilon, \sigma)$-dense if $\left(V_{1}, V_{2}\right)$ is an $(\epsilon, \sigma)$-dense pair.

Now, let us consider the following problem. For a bipartite graph $G$ with $n$ vertices in each color class and density $d$, we want to find an $(\epsilon, d / 2)$-dense pair as large as possible. (The choice of $\sigma=d / 2$ is not essential here.) 
Definition 1.3 For any given $0<\epsilon, d<1$ and a positive integer $n, f(\epsilon, d, n)$ is the largest integer $f$ such that every bipartite graph $G$ with $n$ vertices in each color class and density at least d contains an $(\epsilon, d / 2)$-dense subgraph with $f$ vertices in each color class.

As for $\epsilon \leq 2-\sqrt{2.5}$, every $\epsilon$-regular pair with density at least $(1-\epsilon / 3) d$ is $(\epsilon, d / 2)$ dense, Theorem 1.1 immediately implies that $f(\epsilon, d, n) \geq \frac{n}{2} d^{c / \epsilon^{2}}$.

In 1991, Komlós stated the following lower bound for $f(\epsilon, d, n)$.

Theorem 1.2 [8] For all $0<\epsilon \leq \epsilon_{0}, 0<d<1$ and for all integers $n$,

$$
f(\epsilon, d, n) \geq n d^{(3 / \epsilon) \ln (1 / \epsilon)}
$$

In Section 2 of this paper we prove a different bound which is better for small values of $\epsilon$.

Theorem 1.3 For all $0<\epsilon<1,0<d<1$, and for all integers $n$,

$$
f(\epsilon, d, n) \geq \frac{1}{2} n d^{12 / \epsilon}
$$

We also prove the following upper bound on $f(\epsilon, d, n)$, which shows that, up to a constant, Theorem 1.3 is best possible.

Theorem 1.4 For all $0<\epsilon \leq \epsilon_{0}$ and $0<d \leq d_{0}$, there exists $n_{0}<(1 / d)^{1 /(12 \epsilon)}$ such that for all $n \geq n_{0}$,

$$
f(\epsilon, d, n)<4 n d^{c / \epsilon}
$$

where $c$ is an absolute constant.

In fact, we prove a stronger result than Theorem 1.4.

Definition 1.4 Let $G=\left(V_{1}, V_{2}, E\right)$ be a bipartite graph and $0<\epsilon<1$. A pair $\left(U_{1}, U_{2}\right)$, where $U_{1} \subseteq V_{1}, U_{2} \subseteq V_{2}$, is said to contain an $\epsilon$-hole if there exist $W_{1} \subseteq U_{1}$ and $W_{2} \subseteq U_{2}$ with $\left|W_{1}\right| \geq \epsilon\left|U_{1}\right|$ and $\left|W_{2}\right| \geq \epsilon\left|U_{2}\right|$ such that $e\left(W_{1}, W_{2}\right)=0$.

By definition, if a pair contains an $\epsilon$-hole, then it cannot be $(\epsilon, \sigma)$-dense for any $\sigma>0$.

Definition 1.5 For any given $0<\epsilon, d<1$ and a positive integer $n$, let $h(\epsilon, d, n)$ be the largest integer $h$ such that, every bipartite graph $G$ with $n$ vertices in each color class and density at least $d$ contains a subgraph with h vertices in each color class and with no $\epsilon$-hole. 
Clearly, $f(\epsilon, d, n) \leq h(\epsilon, d, n)$.

Theorem 1.5 For all $0<\epsilon \leq \epsilon_{0}$ and $0<d \leq d_{0}$ there exists $n_{0}<(1 / d)^{1 /(12 \epsilon)}$ such that for all $n \geq n_{0}$,

$$
h(\epsilon, d, n)<4 n d^{c / \epsilon}
$$

where $c$ is an absolute constant.

With no effort to optimize, it follows from the proofs of Theorems 1.4 and 1.5 that the constant $c$ appearing in them can be equal to $1 / 2000$.

\section{Lower bound}

In this section we prove the lower bound given in Theorem 1.3. That is, we show that any bipartite graph $G=\left(V_{1}, V_{2}, E\right)$ with $n$ vertices in each color class and density $d$ contains an $(\epsilon, d / 2)$-dense bipartite subgraph with at least $\frac{1}{2} n d^{c_{1} / \epsilon}$ vertices in each color class. We then show that Theorem 1.1 the proof of which is a refinement of the proof of Theorem 1.3 .

Before giving the proof of Theorem 1.3, we prove the following claim which plays a crucial role.

Claim 2.1 Every bipartite graph $H=\left(V_{1}^{H}, V_{2}^{H}, E\right)$ with $\left|V_{1}^{H}\right|=\left|V_{2}^{H}\right|=m$ contains a pair $\left(U_{1}, U_{2}\right)$ satisfying one of the following conditions:

1. $\left(U_{1}, U_{2}\right)$ is an $(\epsilon, d(H) / 2)$-dense pair and $\left|U_{1}\right|=\left|U_{2}\right| \geq m / 2$,

2. $\left|U_{1}\right|=\left|U_{2}\right| \geq m / 4$ and $d\left(U_{1}, U_{2}\right) \geq(1+\epsilon / 8) d(H)$.

Proof: Assuming that $H$ contains no pair satisfying condition 1, we are going to prove that $H$ contains a pair satisfying condition 2. For simplicity, we assume that $1 / \epsilon$ is an integer.

Since, in particular, $H$ itself is not $(\epsilon, d(H) / 2)$-dense, there exist $A_{1}^{\prime} \subset V_{1}^{H}, B_{1}^{\prime} \subset V_{2}^{H}$ with $\left|A_{1}^{\prime}\right|=\left|B_{1}^{\prime}\right| \geq \epsilon m$ and $e\left(A_{1}^{\prime}, B_{1}^{\prime}\right)<\frac{d(H)}{2}\left|A_{1}^{\prime}\right|\left|B_{1}^{\prime}\right|$. By an averaging argument, we can take $A_{1} \subset A_{1}^{\prime}, B_{1} \subset B_{1}^{\prime}$ satisfying $\left|A_{1}\right|=\left|B_{1}\right|=\frac{\epsilon}{2} m$ and $e\left(A_{1}, B_{1}\right)<\frac{d(H)}{2}\left|A_{1}\right|\left|B_{1}\right|$. (For simplification, we assume that $\frac{\epsilon}{2} m$ is an integer. Later we will make similar assumption which are not essential but simplify our presentation.) Let $F_{1}$ be the graph obtained by removing $A_{1}$ from $V_{1}^{H}$ and $B_{1}$ from $V_{2}^{H}$.

By the assumption, $F_{1}$ is not an $(\epsilon, d(H) / 2)$-dense graph, and we apply the same argument as above to $F_{1}$.

In general, after $l$ steps, $l<1 / \epsilon$, we define $l$ disjoint pairs $\left(A_{1}, B_{1}\right), \cdots,\left(A_{l}, B_{l}\right)$ of size $\left|A_{i}\right|=\left|B_{i}\right|=\frac{\epsilon}{2} m$ for $1 \leq i \leq l$. Assume that $F_{l}$ is obtained by removing $\bigcup_{j=1}^{l} A_{j}$ from $V_{1}^{H}$ and $\bigcup_{j=1}^{l} B_{j}$ from $V_{2}^{H}$. By assumption, $F_{l}$ is not $(\epsilon, d(H) / 2)$-dense, therefore there exists 
$A_{l+1}^{\prime} \subset V_{1}^{H} \backslash \bigcup_{j=1}^{l} A_{j}, B_{l+1}^{\prime} \subset V_{2}^{H} \backslash \bigcup_{j=1}^{l} B_{j}$ of size $\left|A_{l+1}^{\prime}\right|=\left|B_{l+1}^{\prime}\right| \geq \epsilon(1-l \epsilon / 2) m \geq \frac{\epsilon}{2} m$ and $e\left(A_{l+1}^{\prime}, B_{l+1}^{\prime}\right)<\frac{d(H)}{2}\left|A_{l+1}^{\prime}\right|\left|B_{l+1}^{\prime}\right|$. Take $A_{l+1} \subset A_{l+1}^{\prime}, B_{l+1} \subset B_{l+1}^{\prime},\left|A_{l+1}\right|=\left|B_{l+1}\right|=$ $\frac{\epsilon}{2} m$ and $e\left(A_{l+1}, B_{l+1}\right)<\frac{d(H)}{2}\left|A_{l+1}\right|\left|B_{l+1}\right|$.

After $1 / \epsilon$ steps the sets $\bigcup_{j=1}^{1 / \epsilon} A_{j}$ cover a half of $V_{1}^{H}$, and the sets $\bigcup_{j=1}^{1 / \epsilon} B_{j}$ cover a half of $V_{2}^{H}$. Denote $\bar{V}_{1}=\bigcup_{j=1}^{1 / \epsilon} A_{j}$ and $\bar{V}_{2}=\bigcup_{j=1}^{1 / \epsilon} B_{j}$. Set $e_{0}=e\left(\bar{V}_{1}, \bar{V}_{2}\right), e_{1}=$ $e\left(\bar{V}_{1}, V_{2}^{H} \backslash \bar{V}_{2}\right), e_{2}=e\left(V_{1}^{H} \backslash \bar{V}_{1}, \bar{V}_{2}\right), e_{3}=e\left(V_{1}^{H} \backslash \bar{V}_{1}, V_{2}^{H} \backslash \bar{V}_{2}\right)$.

Now we claim that there exists a pair satisfying condition 2. Indeed, if

$$
e_{0} \leq(1-3 \epsilon / 8) d(H) m^{2} / 4
$$

then

$$
e_{1}+e_{2}+e_{3}=d(H) m^{2}-e_{0} \geq 3\left(1+\frac{\epsilon}{8}\right) d(H) \frac{m^{2}}{4} .
$$

Therefore, there exists $i \in\{1,2,3\}$ satisfying

$$
e_{i} \geq\left(1+\frac{\epsilon}{8}\right) d(H) \frac{m^{2}}{4}
$$

and we find a pair satisfying condition 2 .

If $e_{0}>(1-3 \epsilon / 8) d(H) m^{2} / 4$, we define $e_{i j}=e\left(A_{i}, B_{j}\right)$. Then

$\sum_{i} \sum_{j \neq i} e_{i j}=e_{0}-\sum_{i=1}^{1 / \epsilon} e\left(A_{i}, B_{i}\right)>\left(1-\frac{3 \epsilon}{8}\right) d(H) \frac{m^{2}}{4}-\frac{1}{\epsilon} \frac{d(H)}{2}\left(\frac{\epsilon m}{2}\right)^{2}=\left(1-\frac{7}{8} \epsilon\right) d(H) \frac{m^{2}}{4}$.

For any $I \subset\{1, \ldots, 1 / \epsilon\}$ of size $|I|=1 /(2 \epsilon)$, we define

$$
e(I)=\sum_{i \in I} \sum_{j \in\{1, \ldots, 1 / \epsilon\} \backslash I} e_{i j} .
$$

Then $\sum_{I} e(I)$ counts each $e_{i j}$ exactly $\left(\begin{array}{c}1 / \epsilon-2 \\ 1 /(2 \epsilon)-1\end{array}\right)$ times, where $i \neq j$. Thus, there exists $I_{0}$ such that

$$
e\left(I_{0}\right) \geq \frac{\sum_{I} e(I)}{\left(\begin{array}{c}
1 / \epsilon \\
1 /(2 \epsilon)
\end{array}\right)}=\frac{\left(\begin{array}{c}
1 / \epsilon-2 \\
1 /(2 \epsilon)-1
\end{array}\right)}{\left(\begin{array}{c}
1 / \epsilon \\
1 /(2 \epsilon)
\end{array}\right)} \sum_{i} \sum_{j \neq i} e_{i j}>\frac{(1-7 \epsilon / 8) d(H) m^{2} / 4}{4(1-\epsilon)} \geq\left(1+\frac{\epsilon}{8}\right) d(H) \frac{m^{2}}{16},
$$

and consequently the pair $\left(\bigcup_{i \in I_{0}} A_{i}, \bigcup_{j \in\{1, \ldots, 1 / \epsilon\} \backslash I_{0}} B_{j}\right)$ satisfies condition 2.

Proof of Theorem 1.3. Let $G=\left(V_{1}, V_{2}, E\right)$ be any bipartite graph with $n$ vertices in each color class and density $d$. If $G$ contains a pair satisfying condition 1 in Claim 2.1, then we are done. Otherwise, by Claim 2.1, there exists an induced subgraph $G_{1} \subset G$ with at least $n / 4$ vertices in each color class and $d\left(G_{1}\right) \geq(1+\epsilon / 8) d$. Applying Claim 2.1 to $G_{1}$, if $G_{1}$ contains a pair satisfying condition 1 in Claim 2.1, then we have an 
$\left(\epsilon, d\left(G_{1}\right) / 2\right)$-dense pair, which is also an $(\epsilon, d / 2)$-dense pair, with at least $n / 8$ vertices in each color class, and we are done again. Otherwise we find an induced subgraph $G_{2} \subset G_{1}$ with at least $n / 16$ vertices in each color class and $d\left(G_{2}\right) \geq(1+\epsilon / 8)^{2} d$.

Suppose we have iterated this process $s$ times, obtaining a subgraph $G_{s}$ of $G$ with at least $n / 4^{s}$ vertices in each color class and density at least $(1+\epsilon / 8)^{s} d$. If the $(s+1)$-th iteration cannot be completed, it means that $G_{s}$ contains an $(\epsilon, d / 2)$-dense subgraph with at least $n /\left(2 \cdot 4^{s}\right)$ vertices in each color class. Because the density of any graph is not larger than 1 , we can only iterate this process at most $t$ times, where $t$ is the smallest integer such that

$$
\left(1+\frac{\epsilon}{8}\right)^{t+1} d>1
$$

Hence, at some point an $(\epsilon, d / 2)$-dense subgraph with at least $n /\left(2 \cdot 4^{t}\right)$ vertices in each color class must be found. It remains to estimate $t$ from above. By the choice of $t$, we have $(1+\epsilon / 8)^{t} d \leq 1$, or, equivalently,

$$
t \leq \frac{\log _{2}(1 / d)}{\log _{2}(1+\epsilon / 8)},
$$

and so

$$
4^{t}=2^{2 t} \leq(1 / d)^{\frac{2}{\log _{2}(1+\epsilon / 8)}} .
$$

Notice that $\log _{2}(1+\epsilon / 8) \geq \epsilon / 6$ for $0<\epsilon<1$. Indeed, it follows from the facts that $g(x)=\log _{2}(1+\epsilon / 8)-\epsilon / 6$ is concave in $[0,1], g(0)=0$ and $g(1)>0$. Therefore

$$
\frac{1}{2} \frac{n}{4^{t}} \geq \frac{1}{2} n d^{12 / \epsilon},
$$

and consequently we have proved the existence of an $(\epsilon, d / 2)$-dense subgraph of $G$ with at least $\frac{1}{2} n d^{12 / \epsilon}$ vertices in each color class. This completes the proof of Theorem 1.3.

Proof of Theorem 1.1 (Sketch). The proof of Theorem 1.1 is similar to the proof of Theorem 1.3; the only modification is to replace Claim 2.1 by Claim 2.3 below.

The first alternative of Claim 2.3, rather than asking for a large $\epsilon$-regular pair, demands a stronger property which is however easier to analyze.

Definition 2.1 Let $G=\left(V_{1}, V_{2}, E\right)$ be a bipartite graph, $0<\epsilon<1$. A pair $\left(U_{1}, U_{2}\right)$, where $U_{1} \subseteq V_{1}$ and $U_{2} \subseteq V_{2}$, is called $(\epsilon, G)$-regular if for every $W_{1} \subseteq U_{1}$ and $W_{2} \subseteq U_{2}$ with $\left|W_{1}\right| \geq \epsilon\left|U_{1}\right|$ and $\left|W_{2}\right| \geq \epsilon\left|U_{2}\right|$, we have

$$
(1-\epsilon / 3) d(G) \leq d\left(W_{1}, W_{2}\right) \leq(1+\epsilon / 3) d(G) .
$$

Fact 2.2 Every $(\epsilon, G)$-regular pair $\left(U_{1}, U_{2}\right)$ is $\epsilon$-regular. 
Claim 2.3 Every bipartite graph $H=\left(V_{1}^{H}, V_{2}^{H}, E\right)$ with $\left|V_{1}^{H}\right|=\left|V_{2}^{H}\right|=m$ contains a pair $\left(U_{1}, U_{2}\right)$ satisfying one of the following conditions:

1. $\left|U_{1}\right|,\left|U_{2}\right| \geq m / 2$ and $\left(U_{1}, U_{2}\right)$ is $(\epsilon, H)$-regular,

2. $\left|U_{1}\right|,\left|U_{2}\right| \geq m \epsilon / 2$ and $d\left(U_{1}, U_{2}\right) \geq(1+\epsilon / 3) d(H)$,

3. $\left|U_{1}\right|,\left|U_{2}\right| \geq m / 4$ and $d\left(U_{1}, U_{2}\right) \geq\left(1+\epsilon^{2} / 12\right) d(H)$.

Assuming that $H$ contains no pair satisfying conditions 1 or 2 , and using the same technique as in the proof of Claim 2.1, we can prove that $H$ must contain a pair satisfying condition 3.

Applying Claim 2.3, one can prove Theorem 1.1 in the same way as we derived Theorem 1.3 from Claim 2.1 (see the Appendix for details). Note that the obtained $\epsilon$-regular pair $\left(U_{1}, U_{2}\right)$ has density at least $(1-\epsilon / 3) d$.

\section{$3 \quad$ Upper bound}

In this section we prove the upper bound for $h(\epsilon, d, n)$ given in Theorem 1.5. To prove that $h(\epsilon, d, n)<u$, we need to find a bipartite graph $G$ with $n$ vertices in each color class and density at least $d$ such that every subgraph of $G$ with $u$ vertices in each color class contains an $\epsilon$-hole. The following construction will be central for the proof.

Let $k$ and $t$ be positive integers, and $[t]$ denote $\{1,2, \ldots, t\}$. Let $G(k, t)=\left(V_{1}, V_{2}, E\right)$ be the bipartite graph with

$$
\begin{aligned}
& V_{1}=\left\{\mathbf{x}=\left(x_{1}, x_{2}, \ldots, x_{t}\right): 1 \leq x_{s} \leq k, 1 \leq s \leq t .\right\}, \\
& V_{2}=\left\{\mathbf{y}=\left(y_{1}, y_{2}, \ldots, y_{t}\right): 1 \leq y_{s} \leq k, 1 \leq s \leq t .\right\},
\end{aligned}
$$

and $\mathbf{x y} \in E$ if and only if $x_{s} \neq y_{s}$ for each $s \in[t]$, where $\mathbf{x}=\left(x_{1}, x_{2}, \ldots, x_{t}\right) \in V_{1}$ and $\mathbf{y}=\left(y_{1}, y_{2}, \ldots, y_{t}\right) \in V_{2}$.

Observe that $G(k, t)$ is a bipartite graph with $k^{t}$ vertices in each color class and density $\left(\frac{k-1}{k}\right)^{t}$. For $G(k, t)$ we prove the following property. From now on we set $n_{1}=k^{t}$.

Lemma 3.1 Let $k$ and $t$ be positive integers and let $0<\epsilon \leq 1 / 4 k$. For every $U_{1} \subseteq V_{1}$, $U_{2} \subseteq V_{2}$ such that

$$
\min \left\{\left|U_{1}\right|,\left|U_{2}\right|\right\} \geq n_{1}\left(\frac{e}{2 \epsilon k}\right)^{2 \epsilon k t}\left(\frac{1+4 \epsilon k}{\sqrt{2}}\right)^{2 t}
$$

there exists an $\epsilon$-hole in the subgraph of $G(k, t)$ induced by the sets $U_{1}$ and $U_{2}$. 
Proof: $\quad$ Suppose that there is no $\epsilon$-hole in the subgraph of $G(k, t)$ induced by the sets $U_{1}, U_{2}$. We will estimate $\min \left\{\left|U_{1}\right|,\left|U_{2}\right|\right\}$ from above.

For each $s=1,2, \ldots, t$, the integer $i \in[k]$ is called rare with respect to $s$ in $U_{1}$ if

$$
\left|\left\{\mathbf{x} \in U_{1}: x_{s}=i\right\}\right|<\epsilon\left|U_{1}\right| .
$$

Otherwise $i$ is called frequent with respect to $s$. Let $R_{s}^{1}$ be the set of all rare values $i \in[k]$ with respect to $s$ in $U_{1}$ and $F_{s}^{1}$ be the set of all frequent values $i \in[k]$ with respect to $s$ in $U_{1}$. Similarly, let $F_{s}^{2}$ be the set of all frequent values $i \in[k]$ with respect to $s$ in $U_{2}$. Note that $F_{s}^{1} \cap F_{s}^{2}=\emptyset$ for each $s \in[t]$, since otherwise the vertices $\mathbf{x} \in U_{1}$ and $\mathbf{y} \in U_{2}$ with $x_{s}=y_{s}=i \in F_{s}^{1} \cap F_{s}^{2}$ would form an $\epsilon$-hole between $U_{1}$ and $U_{2}$.

Next we are going to prove that more than half of the vertices in $U_{1}$ have each less than $2 \epsilon k$ rare coordinates. At the same time we give an upper bound on the number of such vertices which enables us to estimate $\left|U_{1}\right|$.

For every $\mathbf{x}=\left(x_{1}, \ldots, x_{s}, \ldots, x_{t}\right) \in V_{1}$, define $S_{\mathbf{x}}=\left\{s: x_{s} \in R_{s}^{1}\right\}$. Let $V_{1}^{\prime}=\left\{\mathbf{x} \in V_{1}\right.$ : $\left.\left|S_{\mathbf{x}}\right|<2 \epsilon k t\right\}$ and $U_{1}^{\prime}=U_{1} \cap V_{1}^{\prime}$.

\section{Claim 3.2}

$$
\begin{gathered}
\left|U_{1}^{\prime}\right|>\frac{1}{2}\left|U_{1}\right| \\
\left|U_{1}^{\prime}\right| \leq\left|V_{1}^{\prime}\right| \leq 2 \epsilon k t\left(\frac{e}{2 \epsilon k}\right)^{2 \epsilon k t}\left(\frac{2 \epsilon k^{2} t+\sum_{s=1}^{t}\left|F_{s}^{1}\right|}{t}\right)^{t}
\end{gathered}
$$

Proof of Claim 3.2: To prove (2), we use a standard double counting argument. Consider an auxiliary bipartite graph $M=\left(U_{1},[t], E(M)\right)$ in which a pair $\{\mathbf{x}, s\} \in E(M)$ if and only if $x_{s} \in R_{s}^{1}$, where $\mathbf{x}=\left(x_{1}, x_{2}, \ldots, x_{t}\right) \in U_{1}$ and $s \in[t]$. By the definition of $R_{s}^{1}$, it is easy to see that $\operatorname{deg}_{M}(s)<\epsilon k\left|U_{1}\right|$ for any $s \in[t]$. Therefore there are fewer than $\frac{1}{2}\left|U_{1}\right|$ vertices $\mathbf{x} \in U_{1}$ which satisfy $\left|S_{\mathbf{x}}\right|=\operatorname{deg}_{M}(\mathbf{x}) \geq 2 \epsilon k t$.

Now we prove (3). Let $L \subset[t]$ with $|L|<2 \epsilon k t$. Then by the definition of $S_{\mathbf{x}}$,

$$
\left|\left\{\mathbf{x} \in V_{1}: S_{\mathbf{x}}=L\right\}\right| \leq \prod_{q \in L}\left|R_{q}^{1}\right| \prod_{s \in[t] \backslash L}\left|F_{s}^{1}\right| .
$$

Hence

$$
\left|V_{1}^{\prime}\right| \leq \sum_{L \subset[t],|L|<2 \epsilon k t} k^{|L|} \prod_{s \in[t] \backslash L}\left|F_{s}^{1}\right| .
$$

Since the geometric mean is not larger than the arithmetic mean, we obtain

$$
\left|U_{1}^{\prime}\right| \leq\left|V_{1}^{\prime}\right| \leq \sum_{l<2 \epsilon k t}\left(\begin{array}{l}
t \\
l
\end{array}\right)\left(\frac{k l+\sum_{s=1}^{t}\left|F_{s}^{1}\right|}{t}\right)^{t} .
$$

Since $l<2 \epsilon k t \leq t / 2$, we have $\left(\begin{array}{l}t \\ l\end{array}\right) \leq\left(\begin{array}{c}t \\ 2 \epsilon k t\end{array}\right) \leq\left(\frac{e}{2 \epsilon k}\right)^{2 \epsilon k t}$, and

$$
\left|V_{1}^{\prime}\right| \leq 2 \epsilon k t\left(\frac{e}{2 \epsilon k}\right)^{2 \epsilon k t}\left(\frac{2 \epsilon k^{2} t+\sum_{s=1}^{t}\left|F_{s}^{1}\right|}{t}\right)^{t}
$$


which completes the proof of the claim.

Now we continue the proof of Lemma 3.1. By Claim 3.2

$$
\left|U_{1}\right|<2\left|U_{1}^{\prime}\right| \leq 2\left|V_{1}^{\prime}\right| \leq 4 \epsilon k t\left(\frac{e}{2 \epsilon k}\right)^{2 \epsilon k t}\left(\frac{2 \epsilon k^{2} t+\sum_{s=1}^{t}\left|F_{s}^{1}\right|}{t}\right)^{t} .
$$

Similarly,

$$
\left|U_{2}\right|<4 \epsilon k t\left(\frac{e}{2 \epsilon k}\right)^{2 \epsilon k t}\left(\frac{2 \epsilon k^{2} t+\sum_{s=1}^{t}\left|F_{s}^{2}\right|}{t}\right)^{t} .
$$

Since $F_{s}^{1} \cap F_{s}^{2}=\emptyset$ for each $s \in[t]$, we have $\sum_{s=1}^{t}\left|F_{s}^{1}\right| \leq \frac{t k}{2}$ or $\sum_{s=1}^{t}\left|F_{s}^{2}\right| \leq \frac{t k}{2}$. Therefore,

$$
\begin{aligned}
\min \left\{\left|U_{1}\right|,\left|U_{2}\right|\right\} & <4 \epsilon k t\left(\frac{e}{2 \epsilon k}\right)^{2 \epsilon k t}\left(\frac{2 \epsilon k^{2} t+\frac{k t}{2}}{t}\right)^{t} \\
& =4 \epsilon k t\left(\frac{e}{2 \epsilon k}\right)^{2 \epsilon k t}(1+4 \epsilon k)^{t}\left(\frac{k}{2}\right)^{t} .
\end{aligned}
$$

Applying the inequality $4 \epsilon k t<(1+4 \epsilon k)^{t}$, we finally obtain that

$$
\begin{aligned}
\min \left\{\left|U_{1}\right|,\left|U_{2}\right|\right\} & <\left(\frac{e}{2 \epsilon k}\right)^{2 \epsilon k t}(1+4 \epsilon k)^{2 t}\left(\frac{k}{2}\right)^{t} \\
& =n_{1}\left(\frac{e}{2 \epsilon k}\right)^{2 \epsilon k t}\left(\frac{1+4 \epsilon k}{\sqrt{2}}\right)^{2 t},
\end{aligned}
$$

which completes the proof.

Now for any $n \geq n_{1}$, let $r$ and $q$, where $0 \leq q<n_{1}$, be the positive integers such that $n=r n_{1}+q$. We "blow up" the graph $G(k, t)$ in the following sense: fix any $q$ vertices in each color class, and replace each of them by $r+1$ new vertices. At the same time replace every other vertex by $r$ new vertices. Finally, replace every edge of $G(k, t)$ by the corresponding complete bipartite graph $\left(K_{r, r}, K_{r+1, r}\right.$, or $\left.K_{r+1, r+1}\right)$. Denote this new graph by $G_{n}(k, t)=\left(V_{1}^{n}, V_{2}^{n}, E\right)$. It is easy to see that

$$
\frac{r}{r+1}\left(\frac{k-1}{k}\right)^{t} \leq d\left(G_{n}(k, t)\right) \leq \frac{r+1}{r}\left(\frac{k-1}{k}\right)^{t} .
$$

For this graph we now prove the following lemma which is very similar to Lemma 3.1. Recall that $n_{1}=k^{t}$.

Lemma 3.3 Let $k$ and $t$ be positive integers and let $0<\epsilon \leq 1 / 4 k$. For every $n \geq n_{1}$, and for all $U_{1} \subseteq V_{1}^{n}, U_{2} \subseteq V_{2}^{n}$ such that

$$
\min \left\{\left|U_{1}\right|,\left|U_{2}\right|\right\} \geq 2 n\left(\frac{e}{2 \epsilon k}\right)^{2 \epsilon k t}\left(\frac{1+4 \epsilon k}{\sqrt{2}}\right)^{2 t},
$$

there exists an $\epsilon$-hole in the subgraph of $G_{n}(k, t)$ induced by the sets $U_{1}$ and $U_{2}$. 
Proof: $\quad$ Assume that there is no $\epsilon$-hole in the subgraph of $G_{n}(k, t)$ induced by the sets $U_{1}, U_{2}$. For each $s \in[t]$, define rare and frequent values $i \in[k]$ with respect to $s$, for $U_{1}$ and $U_{2}$, in the same way as in the proof of Lemma 3.1. We follow the lines of the proof of Lemma 3.1. The only novelty is to multiply the right hand side of equations (4) - (11) by $r+1$. Therefore, we have

$$
\min \left\{\left|U_{1}\right|,\left|U_{2}\right|\right\}<(r+1) n_{1}\left(\frac{e}{2 \epsilon k}\right)^{2 \epsilon k t}\left(\frac{1+4 \epsilon k}{\sqrt{2}}\right)^{2 t} .
$$

Since $(r+1) / r \leq 2$, and thus $(r+1) n_{1} \leq 2 r n_{1} \leq 2\left(r n_{1}+q\right)=2 n$, we obtain

$$
\min \left\{\left|U_{1}\right|,\left|U_{2}\right|\right\}<2 n\left(\frac{e}{2 \epsilon k}\right)^{2 \epsilon k t}\left(\frac{1+4 \epsilon k}{\sqrt{2}}\right)^{2 t} .
$$

The goal of blowing up $G(k, t)$ was to obtain graphs with more vertices than $n_{1}$ and still having $\epsilon$-holes in large subgraphs. Next we consider a random "contraction" of $G(k, t)$ to obtain graphs with fewer than $n_{1}$ vertices and with the same property.

From now on, to make our description simpler, we set

$\alpha=\log _{k} \frac{k}{k-1}, \quad \delta=\log _{k} 2-2 \epsilon k \log _{k} \frac{e}{2 \epsilon k}-2 \log _{k}(1+4 \epsilon k), \quad n_{0}=\max \left\{n_{1}^{3 \alpha / 2}, n_{1}^{3 \delta / 2}\right\}$.

Note that $n_{0} \leq n_{1}$ when $k \geq 3$, and under this notation,

$$
n_{1}^{-\alpha}=d(G(k, t))=\left(\frac{k-1}{k}\right)^{t}
$$

and

$$
n_{1}^{-\delta}=\left(\frac{e}{2 \epsilon k}\right)^{2 \epsilon k t}\left(\frac{1+4 \epsilon k}{\sqrt{2}}\right)^{2 t} .
$$

Lemma 3.4 Let $k \geq 3$ be a positive integer, $0<\epsilon \leq 1 / 4 k$, and $t>t_{0}=t_{0}(k, \epsilon)$. Then, for every $n_{0} \leq n<n_{1}$, there exists a graph $G_{n}=\left(V_{1}^{n}, V_{2}^{n}, E^{n}\right)$ with $n$ vertices in each color class such that

$$
\frac{k-1}{k} n_{1}^{-\alpha} \leq d\left(G_{n}\right) \leq \frac{k}{k-1} n_{1}^{-\alpha},
$$

and for all $U_{1} \subseteq V_{1}^{n}, U_{2} \subseteq V_{2}^{n}$ with

$$
\min \left\{\left|U_{1}\right|,\left|U_{2}\right|\right\} \geq 4 n\left(\frac{e}{2 \epsilon k}\right)^{2 \epsilon k t}\left(\frac{1+4 \epsilon k}{\sqrt{2}}\right)^{2 t},
$$

there exists an $\epsilon$-hole in the subgraph of $G_{n}$ induced by the sets $U_{1}$ and $U_{2}$. 
Proof: $\quad$ We define a random subgraph $G^{*}(k, t)=\left(V_{1}^{*}, V_{2}^{*}, E^{*}\right)$ of $G(k, t)$ by choosing uniformly an $n$-element subset $V_{1}^{*}$ of $V_{1}$, and independently, an $n$-element subset $V_{2}^{*}$ of $V_{2}$, and including to $E^{*}$ all edges of $G(k, t)$ with one endpoint in $V_{1}^{*}$ and the other in $V_{2}^{*}$.

For each $v \in V_{1}$, let $N(v)$ denote the neighborhood of $v$ in $G(k, t)$. Then $\left|N(v) \cap V_{2}^{*}\right|$ is a random variable with hypergeometric distribution of expectation $\mathbb{E}\left(\left|N(v) \cap V_{2}^{*}\right|\right)=n n_{1}^{-\alpha}$. Applying Chernoff's inequality ([7], page 27, formula (2.9)),

$$
\operatorname{Prob}\left(\exists v \in V_{1}:|| N(v) \cap V_{2}^{*}\left|-n n_{1}^{-\alpha}\right|>\frac{1}{k} n n_{1}^{-\alpha}\right) \leq 2 n_{1} e^{-n n_{1}^{-\alpha} / 3 k^{2}} .
$$

Define

$$
\mathcal{F}=\left\{\pi=\left(F_{1}, \ldots, F_{t}\right) \text { where } F_{i} \subset[k], \quad i=1, \ldots, t\right\} .
$$

Clearly, $|\mathcal{F}|=2^{k t}=n_{1}^{k \ln 2 / \ln k}$. For every $\pi \in \mathcal{F}$ and $\mathbf{x}=\left(x_{1}, \ldots, x_{s}, \ldots, x_{t}\right) \in V_{i}, i=1,2$, define $S_{\mathbf{x}}^{\pi}=\left\{s: x_{s} \in[k] \backslash F_{s}\right\}$ and $V_{i}(\pi)=\left\{\mathbf{x}:\left|S_{\mathbf{x}}^{\pi}\right|<2 \epsilon k t\right\}$.

For each $\pi \in \mathcal{F}$, and $i=1,2,\left|V_{i}(\pi) \cap V_{i}^{*}\right|$ is a random variable with hypergeometric distribution. If $\left|V_{i}(\pi)\right|<n_{1}^{1-\delta}$, then

$$
\mathbb{E}\left(\left|V_{i}(\pi) \cap V_{i}^{*}\right|\right)=\frac{n}{n_{1}}\left|V_{i}(\pi)\right|<n n_{1}^{-\delta}
$$

Therefore, by Chernoff's inequality (([7], page 28, formula (2.10)),

$$
\operatorname{Prob}\left(\exists \pi \in \mathcal{F} \text { and } \exists i \in\{1,2\}:\left|V_{i}(\pi)\right|<n_{1}^{1-\delta} \text { but }\left|V_{i}(\pi) \cap V_{i}^{*}\right|>2 n n_{1}^{-\delta}\right) \leq \frac{2 n_{1}^{k \ln 2 / \ln k}}{e^{c^{\prime} n n_{1}^{-\delta}}}
$$

where $c^{\prime}=\ln 2-1 / 2$.

Since $n n_{1}^{-\delta} \geq \max \left\{n^{\delta / 2}, n^{\alpha / 2}\right\}$ and $\delta, \alpha$ do not depend on $t$, for sufficiently large $t$ the right hand side of (16) and (18) are each smaller than $1 / 2$, yielding the existence of an induced subgraph $G_{n}=G(k, t)\left[V_{1}^{n}, V_{2}^{n}\right]$ of $G(k, t)$ with $\left|V_{1}^{n}\right|=\left|V_{2}^{n}\right|=n$, which satisfies (15) and such that

$$
\forall \pi \in \mathcal{F}, i=1,2:\left|V_{i}(\pi)\right| \geq n_{1}^{1-\delta} \text { or }\left|V_{i}(\pi) \cap V_{i}^{n}\right| \leq 2 n n_{1}^{-\delta} .
$$

Now take any $U_{1} \subset V_{1}^{n}, U_{2} \subset V_{2}^{n}$ with no $\epsilon$-hole between $U_{1}$ and $U_{2}$. These two sets determine, as in the proof of Lemma 3.1, two sequences $\pi^{1}$ and $\pi^{2}$ of sets of frequent values $F_{s}^{1}$ and $F_{s}^{2}$ such that $F_{s}^{1} \cap F_{s}^{2}=\emptyset, s=1, \ldots t$. Let $U_{i}^{\prime}=\left|V_{i}\left(\pi^{i}\right) \cap V_{i}^{n}\right|$ be defined as in the proof of Lemma 3.1. Then, as it was shown in that proof, $\left|U_{i}\right|<2\left|U_{i}^{\prime}\right|$, and

$$
\min \left\{\left|V_{1}\left(\pi^{1}\right)\right|,\left|V_{2}\left(\pi^{2}\right)\right|\right\}<n_{1}^{1-\delta} .
$$

Hence, by (19),

$$
\begin{aligned}
\left|U_{i}\right| & <2\left|U_{i}^{\prime}\right|=2\left|V_{i}\left(\pi^{i}\right) \cap V_{i}^{n}\right| \leq 4 n n_{1}^{-\delta} \\
& =4 n\left(\frac{e}{2 \epsilon k}\right)^{2 \epsilon k t}\left(\frac{1+4 \epsilon k}{\sqrt{2}}\right)^{2 t}
\end{aligned}
$$


for $i=1$ or $i=2$, a contradiction.

Now we are ready to prove Theorem 1.5.

Proof of Theorem 1.5: Fix any $0<\epsilon \leq \epsilon_{0}$, and let $k \geq 3$ be the integer such that

$$
\frac{1}{25(k+1)}<\epsilon \leq \frac{1}{25 k} \text {. }
$$

Fix any $0<d \leq d_{0} \leq 1 / 8$, and let $t$ be the integer such that

$$
\frac{1}{2}\left(\frac{k-1}{k}\right)^{t+1}<d \leq \frac{1}{2}\left(\frac{k-1}{k}\right)^{t} .
$$

Observe that $k \leq t$, since otherwise

$$
\frac{1}{2}\left(\frac{k-1}{k}\right)^{t+1} \geq \frac{1}{2}\left(\frac{k-1}{k}\right)^{k} \geq \frac{1}{8} \geq d,
$$

a contradiction.

Now recall that $n_{0}=\max \left\{n_{1}^{3 \delta / 2}, n_{1}^{3 \alpha / 2}\right\}$ and consider two separate cases.

Case 1. $n \geq n_{1}=k^{t}$

Take the blown-up graph $G_{n}(k, t)$. By $(13)$, we have

$$
d\left(G_{n}(k, t)\right) \geq \frac{r}{r+1}\left(\frac{k-1}{k}\right)^{t} \geq \frac{1}{2}\left(\frac{k-1}{k}\right)^{t} \geq d .
$$

Thus, by Lemma 3.3

$$
h(\epsilon, d, n)<2 n\left(\frac{e}{2 \epsilon k}\right)^{2 \epsilon k t}\left(\frac{1+4 \epsilon k}{\sqrt{2}}\right)^{2 t} .
$$

Case 2. $n_{0} \leq n<n_{1}$

Take the graph $G_{n}$ satisfying Lemma 3.4. By (15), we have, again,

$$
d\left(G_{n}\right)>\frac{k-1}{k} n_{1}^{-\alpha} \geq d
$$

Thus, by Lemma 3.3

$$
h(\epsilon, d, n)<4 n\left(\frac{e}{2 \epsilon k}\right)^{2 \epsilon k t}\left(\frac{1+4 \epsilon k}{\sqrt{2}}\right)^{2 t} .
$$

Combining these two cases, we conclude that (23) holds for every $n \geq n_{0}$. By reshaping the right hand side of (23), we arrive at

$$
\begin{aligned}
h(\epsilon, d, n) & <4 n\left(\frac{1}{2}\left(\frac{k-1}{k}\right)^{t+1}\right)^{\phi} \\
& <4 n d^{\phi}
\end{aligned}
$$


where

$$
\phi=\frac{t\left(\ln 2-2 \epsilon k \ln \frac{e}{2 \epsilon k}-2 \ln (1+4 \epsilon k)\right)}{\ln 2+(t+1) \ln \frac{k}{k-1}} .
$$

In what follows we will be relying on (20) and the well-known inequalities

$$
x / 2 \leq \ln (1+x) \leq x
$$

valid for $0 \leq x \leq 1$. First notice that

$$
\ln \frac{k}{k-1} \leq \frac{1}{k-1} \leq \frac{3}{k+1}<75 \epsilon
$$

and

$$
\ln 2+(t+1) \ln \frac{k}{k-1} \leq 1+75(t+1) \epsilon<100(t+1) \epsilon
$$

Also

$$
\ln 2-2 \epsilon k \ln \frac{e}{2 \epsilon k}-2 \ln (1+4 \epsilon k)>\frac{1}{10}
$$

when $\epsilon \leq 1 / 25 k$. Indeed, $q(x)=\ln 2-x \ln \frac{e}{x}-2 \ln (1+2 x)$ is decreasing when $x<1$ and $q(2 / 25)>1 / 10$.

Combining (25), (26), (29), (30) and the fact that $t /(t+1) \geq 1 / 2$, we have

$$
h(\epsilon, d, n)<4 n d^{1 / 2000 \epsilon} .
$$

It remains to estimate $n_{0}=\max \left\{n_{1}^{3 \delta / 2}, n_{1}^{3 \alpha / 2}\right\}$. Observe that $n_{1}^{\delta}=\left(\frac{2 \epsilon k}{e}\right)^{2 \epsilon k t}\left(\frac{\sqrt{2}}{1+4 \epsilon k}\right)^{2 t}$ and $n_{1}^{\alpha}=\left(\frac{k}{k-1}\right)^{t}$. We have

$$
\begin{aligned}
n_{1}^{3 \delta / 2} & =\left(\frac{2 \epsilon k}{e}\right)^{3 \epsilon k t}\left(\frac{\sqrt{2}}{1+4 \epsilon k}\right)^{3 t} \\
& =\left[2\left(\frac{k}{k-1}\right)^{t}\right]^{\eta} \\
& \leq(1 / d)^{\eta}
\end{aligned}
$$

where

$$
\eta=\frac{3 t\left[\ln 2-2 \epsilon k \ln \frac{e}{2 \epsilon k}-2 \ln (1+4 \epsilon k)\right]}{2 t \ln \frac{k}{k-1}+2 \ln 2} .
$$

Applying (27) and (20), we have

$$
\eta<\frac{3 \ln 2}{2 \ln (k / k-1)}<3 k \ln 2 \leq \frac{1}{12 \epsilon}
$$


So, by (34), we obtain

$$
n_{1}^{3 \delta / 2}<(1 / d)^{1 /(12 \epsilon)}
$$

We also have

$$
n_{1}^{3 \alpha / 2}=\left(\frac{k}{k-1}\right)^{3 t / 2}<\left[2\left(\frac{k}{k-1}\right)^{t}\right]^{3 / 2} \leq(1 / d)^{3 / 2} .
$$

Comparing (35) and (36), it is easy to see that $n_{1}^{3 \delta / 2} \geq n_{1}^{3 \alpha / 2}$, since $1 /(12 \epsilon) \geq 3 / 2$ when $\epsilon \leq 1 / 50$. Hence, $n_{0}<(1 / d)^{1 /(12 \epsilon)}$.

\section{Applications}

As an immediate application of our Theorem 1.3, we improve slightly an upper bound on the cycle partition number of an $r$-edge-colored $K_{n, n}$ discussed in [6]. The cycle partition number of an $r$-edge-colored graph $G$ is defined to be the minimum $k$ such that whenever the edges of $G$ are colored with $r$ colors, the vertices of $G$ can be covered by at most $k$ vertex-disjoint monochromatic cycles. Erdös, Gyárfás, and Pyber ([3]) proved that the cycle partition number of $r$-colored complete graphs $K^{n}$ is $O\left(r^{2} \ln r\right)$. They also raised the question whether the cycle partition number for the complete bipartite graph $K_{n, n}$ is independent of $n$. In [6], Haxell proved that the upper bound on the cycle partition number for an $r$-edge-colored $K_{n, n}$ is $O\left((r \ln r)^{2}\right)$ ([6]). Replacing Lemma 2 from [6] by our Theorem 1.3, this can be improved to $O\left(r^{2} \ln r\right)$. We omit the details.

We conclude the paper with another application leading to what we believe is an interesting problem. Let $\mathcal{B}(m, \Delta)$ be the family of all bipartite graphs with $m$ vertices in each color class and maximum degree at most $\Delta$. We say that a graph $G$ is $(m, \Delta)$ universal if $G$ contains a copy of $H$ for every $H \in \mathcal{B}(m, \Delta)$. In [1] and [2] the problem of finding minimum $M=M(m)$ for which there exists an $(m, \Delta)$-universal graph with $M$ edges is investigated. Here we apply Theorems 1.3 and 1.4 to a related problem.

Given $\Delta \geq 1,0<d<1$ and $n$, let $g(\Delta, d, n)$ be the largest integer $m$ such that every bipartite graph $G$ with $n$ vertices in each color class and at least $d n^{2}$ edges is $(m, \Delta)$-universal.

Proposition 4.1 For all $\Delta \geq \Delta_{0}$ and $d \leq d_{0}$, there is $n_{0}$ such that for all $n \geq n_{0}$,

$$
\frac{1}{2} n d^{c_{1}(d / 2)^{-\Delta}} \leq g(\Delta, d, n) \leq 4 n d^{c_{2} \Delta / \ln \Delta},
$$

where $c_{1}$ and $c_{2}$ are absolute constants.

Proof: $\quad$ For the proof of the upper bound we need to find, for every $n \geq n_{0}$, a bipartite graph $G$ with $n$ vertices in each color class and $d(G) \geq d$, as well as a graph $H_{0} \in \mathcal{B}(m, \Delta)$ 
such that $H_{0} \nsubseteq \subseteq$. As $G$ we will use the graph considered in the proof of Theorem 1.5 which is known to contain an $\epsilon$-hole in every $m$ by $m$ subgraph, where $m=4 n d^{c / \epsilon}$.

With this approach, a natural candidate for $H_{0}$ is then a graph with no large holes. By considering a random bipartite graph with $2 m$ vertices in each color class and with edge probability $\Delta /(4 m)$, a standard application of the first moment method yields the existence of a graph $H_{0} \in \mathcal{B}(m, \Delta)$ which contains no $9 \ln \Delta / \Delta$-hole. Setting $\epsilon=9 \ln \Delta / \Delta$, this proves the upper bound with $c_{2}=c / 9$, where $c$ is the constant appearing in Theorem 1.5 .

For the lower bound, in addition to Theorem 1.3, we use the following embedding result.

Lemma 4.2 ([5], Lemma 2) Every bipartite, $\left(\sigma^{\Delta}, \sigma\right)$-dense graph $F$ with at least $\sigma^{-{ }^{\Delta}} m$ vertices in each color class is $(m, \Delta)$-universal.

Given $\Delta, d$ and $n$, set $\epsilon=(d / 2)^{\Delta}$ and

$$
m=\frac{1}{2} n(d / 2)^{\Delta} d^{12 / \epsilon} \geq \frac{1}{2} n d^{14 / \epsilon} .
$$

By Theorem 1.3, every bipartite graph $G$ with $n$ vertices in each color class and at least $d n^{2}$ edges contains an $(\epsilon, d / 2)$-dense subgraph $F$ with at least $\frac{1}{2} n d^{12 / \epsilon}=(d / 2)^{-\Delta} m$ vertices in each color class. By Lemma 4.2 with $\sigma=d / 2, F$ is $(m, \Delta)$-universal and so is $G$. This proves the lower bound with $c_{1}=14$.

It seems to be a challenging problem to narrow the gap between the lower and upper bound in Proposition 4.1. We believe that the upper bound is closer to the true value of $g(\Delta, d, n)$. The proof of this fact, however, would require an essential strengthening of the current graph embedding methods.

It is interesting to note that the nonbipartite version of graph $G(k, t)$ which serves as a basis for constructing a counterexample in Theorem 1.5, and consequently in the right hand side of Proposition 4.1, was proved in [1] to be $\left(k^{t}, \Delta\right)$-universal if only $k=k(\Delta)$ is sufficiently large.

Acknowledgment. We thank Andrzej Dudek and an anonymous referee for careful reading of the manuscript.

\section{References}

[1] N. Alon, M. Capalbo, Y. Kohayakawa, V. Rödl, A. Ruciński, E. Szemerédi, Universality and tolerance, In Proceedings of the 41st IEEE Annual Symposium on FOCS (2000), 14-21.

[2] N. Alon, M. Capalbo, Y. Kohayakawa, V. Rödl, A. Ruciński, E. Szemerédi, Nearoptimum universal graphs for graphs with bounded degrees, APPROX-RANDOM 2001, LNCS 3139 (2001) 170-180 
[3] P. Erdös, A. Gyárfás, and L. Pyber, Vertex coverings by monochromatic cycles and trees, J. Combin. Theory Ser. B 51 (1991), 90-95.

[4] W. T. Gowers, Lower bounds of tower type for Szemerédi's uniformity lemma, GAFA, Geom. Funct. Anal. 7 (1997), 322-337.

[5] R. L. Graham, V. Rödl and A. Ruciński, On bipartite graphs with linear Ramsey numbers, Combinatorica, 21 (2001), 199-209.

[6] P. E. Haxell, Partitioning complete bipartite graphs by monochromatic cycles, Journal of Combinatorial Theory, Series B 69, (1997), 210-218.

[7] S. Janson, T. Łuczak, A. Ruciński, Random Graphs, John Wiley and Sons, New York, 2000.

[8] J. Komlós, M.Simonovits, Szemerédi's regularity lemma and its applications in graph theory, Combinatorics, Paul Erdős is Eighty (Volume 2), Keszthely (Hungary), 1993, Budapest (1996), 295-352.

[9] E. Szemerédi, Regular partitions of graphs in Problèmes combinatoires et théorie des graphes, Orsay 1976, J.-C. Bermond, J.-C. Fournier, M. Las Vergnas, D. Sotteau, eds., Colloq. Internat. CNRS 260, Paris, 1978, 399-401.

\section{Appendix}

At the end of Section 2, we sketched how to prove Theorem 1.1. Here we give all the details of that proof.

Proof of Claim 2.3. Assuming that $H$ contains no pair satisfying conditions 1 or 2, we will prove that $H$ must contain a pair satisfying condition 3.

Since, in particular, the pair $\left(V_{1}^{H}, V_{2}^{H}\right)$ is not $(\epsilon, H)$-regular, there exist $A_{1}^{\prime} \subset V_{1}^{H}, B_{1}^{\prime} \subset$ $V_{2}^{H}$ with $\left|A_{1}^{\prime}\right|=\left|B_{1}^{\prime}\right| \geq \epsilon m$ satisfying either

$$
d\left(A_{1}^{\prime}, B_{1}^{\prime}\right)>(1+\epsilon / 3) d(H)
$$

or

$$
d\left(A_{1}^{\prime}, B_{1}^{\prime}\right)<(1-\epsilon / 3) d(H)
$$

If (37) holds, then we have a pair satisfying condition 2. So (38) holds, and by an averaging argument, we can take $A_{1} \subset A_{1}^{\prime}, B_{1} \subset B_{1}^{\prime}$ satisfying $\left|A_{1}\right|=\left|B_{1}\right|=\frac{\epsilon}{2} m$ and $d\left(A_{1}, B_{1}\right) \leq d\left(A_{1}^{\prime}, B_{1}^{\prime}\right)<(1-\epsilon / 3) d(H)$. Let $F_{1}$ be the graph obtained by removing $A_{1}$ from $V_{1}^{H}$ and $B_{1}$ from $V_{2}^{H}$.

We apply the same argument to $F_{1}$, and in general, after $l$ steps, $l<1 / \epsilon$, we define $l$ disjoint pairs $\left(A_{1}, B_{1}\right), \cdots,\left(A_{l}, B_{l}\right)$ of size $\left|A_{i}\right|=\left|B_{i}\right|=\frac{\epsilon}{2} m$ such that $d\left(A_{i}, B_{i}\right)<$ $(1-\epsilon / 3) d(H), 1 \leq i \leq l$. Assume that $F_{l}$ is obtained by removing $\bigcup_{j=1}^{l} A_{j}$ from $V_{1}^{H}$ 
and $\bigcup_{j=1}^{l} B_{j}$ from $V_{2}^{H}$. By our assumption that $H$ does not contain a pair satisfying conditions 1 or 2 , there exist $A_{l+1}^{\prime} \subset V_{1}^{H} \backslash \bigcup_{j=1}^{l} A_{j}, B_{l+1}^{\prime} \subset V_{2}^{H} \backslash \bigcup_{j=1}^{l} B_{j}$ of size $\left|A_{l+1}^{\prime}\right|=$ $\left|B_{l+1}^{\prime}\right| \geq \epsilon(1-l \epsilon / 2) m \geq \frac{\epsilon}{2} m$ satisfying $d\left(A_{l+1}^{\prime}, B_{l+1}^{\prime}\right)<(1-\epsilon / 3) d(H)$, and again we can find $A_{l+1} \subset A_{l+1}^{\prime}$ such that $B_{l+1} \subset B_{l+1}^{\prime},\left|A_{l+1}\right|=\left|B_{l+1}\right|=\frac{\epsilon}{2} m$ and $d\left(A_{l+1}, B_{l+1}\right)<$ $(1-\epsilon / 3) d(H)$.

After $1 / \epsilon$ steps the sets $\bigcup_{j=1}^{1 / \epsilon} A_{j}$ cover a half of $V_{1}^{H}$, and the sets $\bigcup_{j=1}^{1 / \epsilon} B_{j}$ cover a half of $V_{2}^{H}$. Set $\bar{V}_{1}=\bigcup_{j=1}^{1 / \epsilon} A_{j}, \bar{V}_{2}=\bigcup_{j=1}^{1 / \epsilon} B_{j}, e_{0}=e\left(\bar{V}_{1}, \bar{V}_{2}\right), e_{1}=e\left(\bar{V}_{1}, V_{2}^{H} \backslash \bar{V}_{2}\right), e_{2}=$ $e\left(V_{1}^{H} \backslash \bar{V}_{1}, \bar{V}_{2}\right)$, and $e_{3}=e\left(V_{1}^{H} \backslash \bar{V}_{1}, V_{2}^{H} \backslash \bar{V}_{2}\right)$.

We claim that there exists a pair $\left(U_{1}, U_{2}\right)$ satisfying condition 3. Indeed, if

$$
e_{0} \leq\left(1-\epsilon^{2} / 4\right) d(H) m^{2} / 4
$$

then

$$
e_{1}+e_{2}+e_{3}=d(H) m^{2}-e_{0} \geq 3\left(1+\frac{\epsilon^{2}}{12}\right) d(H) \frac{m^{2}}{4},
$$

and therefore, there exists $i \in\{1,2,3\}$ such that

$$
e_{i} \geq\left(1+\frac{\epsilon^{2}}{12}\right) d(H) \frac{m^{2}}{4}
$$

Let $\left(U_{1}, U_{2}\right)$ be the pair defining $e_{i}$. It is easy to see that $\left(U_{1}, U_{2}\right)$ satisfies condition 3 .

If $e_{0}>\left(1-\epsilon^{2} / 4\right) d(H) m^{2} / 4$, we define $e_{i j}=e\left(A_{i}, B_{j}\right)$. By the choice of pairs $\left(A_{i}, B_{i}\right)$, we have $e_{i i}<\left(1-\frac{\epsilon}{3}\right) d(H)\left(\frac{\epsilon m}{2}\right)^{2}$ for every $i \leq 1 / \epsilon$. Therefore

$$
\begin{aligned}
\sum_{i} \sum_{j \neq i} e_{i j}=e_{0}-\sum_{i=1}^{1 / \epsilon} e\left(A_{i}, B_{i}\right) & >\left(1-\frac{\epsilon^{2}}{4}\right) d(H) \frac{m^{2}}{4}-\frac{1}{\epsilon}\left(1-\frac{\epsilon}{3}\right) d(H)\left(\frac{\epsilon m}{2}\right)^{2} \\
& >\left(1-\epsilon+\frac{\epsilon^{2}}{12}\right) d(H) \frac{m^{2}}{4} .
\end{aligned}
$$

For any $I \subset\{1, \ldots, 1 / \epsilon\},|I|=1 /(2 \epsilon)$, we define

$$
e(I)=\sum_{i \in I} \sum_{j \in\{1, \ldots, 1 / \epsilon\} \backslash I} e_{i j}
$$

Then $\sum_{I} e(I)$ counts each $e_{i j}\left(\begin{array}{c}1 / \epsilon-2 \\ 1 /(2 \epsilon)-1\end{array}\right)$ times, where $i \neq j$. Therefore, there exists $I$ such that

$$
e(I) \geq \frac{\sum_{I} e(I)}{\left(\begin{array}{c}
1 / \epsilon \\
1 /(2 \epsilon)
\end{array}\right)}=\frac{\left(\begin{array}{c}
1 / \epsilon-2 \\
1 /(2 \epsilon)-1
\end{array}\right)}{\left(\begin{array}{c}
1 / \epsilon \\
1 /(2 \epsilon)
\end{array}\right)} \sum_{i} \sum_{j \neq i} e_{i j}>\frac{\left(1-\epsilon+\frac{\epsilon^{2}}{12}\right) d m^{2} / 4}{4(1-\epsilon)} \geq\left(1+\frac{\epsilon^{2}}{12}\right) d(H) \frac{m^{2}}{16}
$$

Set $U_{1}=\bigcup_{i \in I} A_{i}, U_{2}=\bigcup_{j \notin I} B_{j}$. Then $\left(U_{1}, U_{2}\right)$ is a pair satisfying condition 3 . 
Proof of Theorem 1.1. Let $G=\left(V_{1}, V_{2}, E\right)$ be a bipartite graph with $\left|V_{1}\right|=\left|V_{2}\right|=n$ and density $d$. If $G$ contains a pair $\left(U_{1}, U_{2}\right)$ satisfying condition 1 in Claim 2.3, then, due to Fact $2.2,\left(U_{1}, U_{2}\right)$ is an $\epsilon$-regular pair with $\left|U_{1}\right|=\left|U_{2}\right| \geq n / 2$.

Assuming that $G$ contains no pair satisfying condition 1 in Claim 2.3, and applying Claim 2.3 to $G$, we can find either a subgraph $G_{(1,0)} \subset G$ with at least $\epsilon n / 2$ vertices in each color class and density at least $(1+\epsilon / 3) d$, or a subgraph $G_{(0,1)} \subset G$ with at least $n / 4$ vertices in each color class and density at least $\left(1+\epsilon^{2} / 12\right) d$.

Without loss of generality, we may assume that the former is true. If $G_{(1,0)}$ contains a pair satisfying condition 1 in Claim 2.3, then this pair is $\epsilon$-regular. So, again, assuming that $G_{(1,0)}$ contains no pair satisfying condition 1 in Claim 2.3, and applying Claim 2.3 to $G_{(1,0)}$, we can find either a subgraph $G_{(2,0)}$ of $G_{(1,0)}$ with at least $n(\epsilon / 2)^{2}$ vertices in each color class and density at least $(1+\epsilon / 3)^{2} d$, or a subgraph $G_{(1,1)}$ of $G_{(1,0)}$ with at least $n \epsilon / 8$ vertices in each color class and density at least $(1+\epsilon / 3)\left(1+\epsilon^{2} / 12\right) d$.

Suppose we have iterated this process $\left(s_{1}, s_{2}\right)$ times, where $s_{1}$ is the number of times of obtaining pairs satisfying condition 2 in Claim 2.3, and $s_{2}$ is the number of times obtaining pairs satisfying condition 3 in Claim 2.3. Then we obtain a subgraph $G_{\left(s_{1}, s_{2}\right)}$ of $G$ with at least $n(\epsilon / 2)^{s_{1}}(1 / 4)^{s_{2}}$ vertices in each color class and density at least $(1+\epsilon / 4)^{s_{1}}(1+$ $\left.\epsilon^{2} / 12\right)^{s_{2}} d$. Because the density of no graph is larger than 1 , this process has to stop in finite times. Let $\left(t_{1}, t_{2}\right)$ be the number of times we iterate before the process stops, then $(1+\epsilon / 3)^{t_{1}}\left(1+\epsilon^{2} / 12\right)^{t_{2}} d \leq 1$.

At this point, we obtain an $\epsilon$-regular pair with at least $\frac{n}{2}(\epsilon / 2)^{t_{1}}(1 / 4)^{t_{2}}$ vertices in each color class. It remains to estimate $t_{1}$ and $t_{2}$ from above. By the choice of $t_{1}, t_{2}$, we have $(1+\epsilon / 3)^{t_{1}} d \leq 1$, and $\left(1+\epsilon^{2} / 12\right)^{t_{2}} d \leq 1$, thus

$$
t_{1} \leq \frac{\ln (1 / d)}{\ln (1+\epsilon / 3)}
$$

and

$$
t_{2} \leq \frac{\ln (1 / d)}{\ln \left(1+\epsilon^{2} / 12\right)}
$$

Hence,

where

$$
\frac{n}{2}(\epsilon / 2)^{t_{1}}(1 / 4)^{t_{2}} \geq \frac{n}{2} d^{\phi} .
$$

$$
\phi=\frac{\ln (2 / \epsilon)}{\ln (1+\epsilon / 3)}+\frac{\ln 4}{\ln \left(1+\epsilon^{2} / 12\right)} .
$$

Notice that $\ln (1+x) \geq x / 2$ holds for $0 \leq x \leq 1$. Therefore

$$
\phi \leq \frac{6 \ln (2 / \epsilon)}{\epsilon}+\frac{48 \ln 2}{\epsilon^{2}} \leq \frac{c}{\epsilon^{2}},
$$

where $c$ is an absolute constant. Applying (40) to (39), we have

$$
\frac{n}{2}(\epsilon / 2)^{t_{1}}(1 / 4)^{t_{2}} \geq \frac{n}{2} d^{c / \epsilon^{2}},
$$

and consequently we have proved the existence of an $\epsilon$-regular pair in $G$ with at least $\frac{1}{2} n d^{c / \epsilon^{2}}$ vertices in each color class. This completes the proof of Theorem 1.1. 